\title{
A Fast and Efficient Shape Descriptor for an Advanced Weed Type Classification Approach
}

\author{
Adil Tannouche, Khalid Sbai, Miloud Rahmoune, Amine Zoubir, Rachid Agounoune, \\ Rachid Saadani, Abdelali Rahmani \\ Laboratoire d'Etude des Matériaux Avancés et Applications, FS-EST, Moulay Ismail University,
} BP 11201, Zitoune, Meknes, Morocco

\section{Article Info}

Article history:

Received Jan 2, 2016

Revised Mar 11, 2016

Accepted Mar 26, 2016

Keyword:

Machine Vision

Real-time Image Processing

Weed Type Classification

Precision Agriculture

Shape Descriptor

\begin{abstract}
In weed management, the distinction between monocots and dicots species is an important issue. Indeed, the yield is much higher with the application of a selective treatment instead of using a broadcast herbicide overall the parcel. This article presents a fast shape descriptor designed to distinguish between these two families of weeds. The efficiency of the descriptor is evaluated by analyzing data with the pattern recognition process known as the discriminant factor analysis (DFA). Excellent results have been obtained in the differentiation between these two weed species.
\end{abstract}

Copyright (c) 2016 Institute of Advanced Engineering and Science. All rights reserved.

\section{Corresponding Author:}

Adil Tannouche,

Departement of Electrical and Computer Engineering,

LEM2A, High School of Technology,

Meknes, Morocco, +212 650960207.

Email: tannouche@gmail.com

\section{INTRODUCTION}

The weed control is a key element in smart agriculture. The chemical weed control is widely practiced in order to reduce the infestation rates and to improve harvests. Weeds are divided into two big families: the Monocotyledonous family which is characterized by long and thin leaves; and the Dicotyledonous family with short leaves (see Figure 1). In view of this diversity, the weed control performance is even better with the application of a selective treatment instead of using a single broadcast herbicide overall the parcel.In this regard, recent researches in computer vision have given birth to several efficient techniques for detection and / or classification of weeds. Traditionally, two main approaches are used:

- A spectral approach : this approach consists in decoding the spectral information to detect the presence of weed in the parcels. In [1] and [2], respectively, the authors used the information revealed by the near infrared (NIR) and the simple (RGB) pictures to detect weeds. In [3], the authors used the UV fluorescence spectrum. In [4], the authors analyzed the hyperspectral images to properly detect and select the weeds.Despite his performance, this technique requires an expensive equipment.

- A spatial approach: this technique focuses on the distribution of weeds in the parcel or on their morphological forms [5] in order to identify them. In [6] and [7], the authors detected weeds by observing their presence in the seed line spacing. In [8], the authors examined the shape of weeds by using the seven moments of $\mathrm{Hu}$ [9] and six shape descriptors to achieve a better selection of weeds. The results are very satisfactory in spite of the processing time of about two frames per second. 
In this study, we present a new innovative, fast and efficient approach for the selection of weeds species. Our approach based essentially by the application of binary descriptor designed for this purpose. This descriptor called Adjacencies Descriptor returns the number of horizontal, vertical and diagonal adjacencies for a given 2D object. This technique allow distinguishing between the shapes with a rounded morphology (Dicot) and others with a long thin morphology (Monocot).
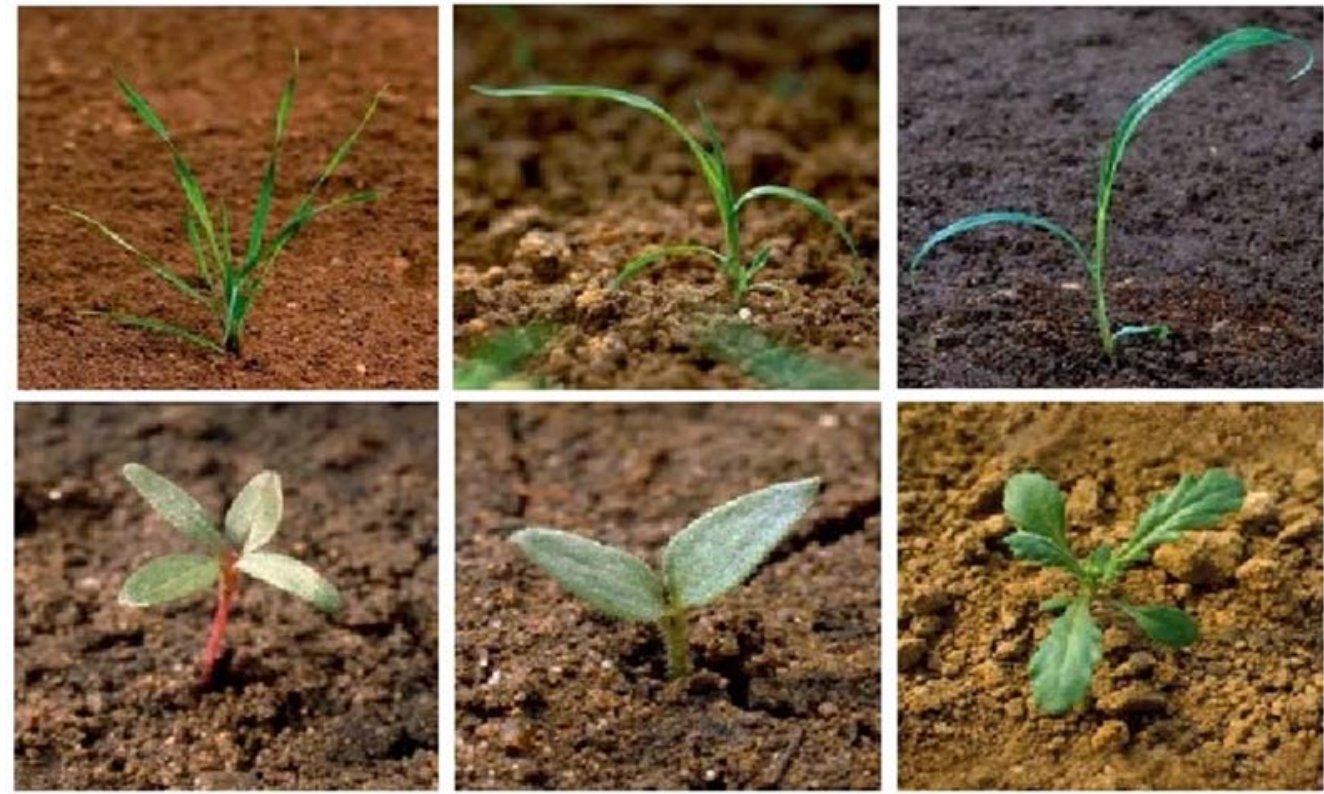

Figure 1. Weed samples: monocot in first line and dicot in second line

\section{MATERIAL AND METHOD}

The imaging system is composed of a standard RGB camera. The camera held in vertical position at 25 to $30 \mathrm{~cm}$ above the region of interest. Thus, the visible scene covers an area of $50 \times 50 \mathrm{~cm}^{2}$ (see Figure 2). This setup allows us to overcome the perspective view problem and improve the spatial resolution.

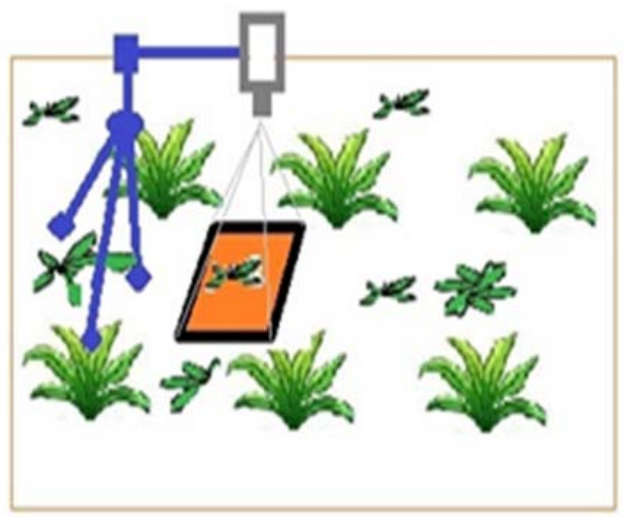

Figure 2. Acquisition process: camera on a tripod at approximately $0.3 \mathrm{~m}$ height pointing vertically downward

\subsection{The Adjacencies Descriptor}

Our region-based descriptor calculates the number of horizontal, vertical and diagonal adjacencies between a given original pixel and their adjacent ones (see Figure 3). The original pixel (green cell), is surrounded by eight other peripheral pixels (yellow and blue cells).

Cells in yellow show horizontal and vertical adjacencies and the blue ones show diagonally adjacencies with respect to the original cell.

A Fast and Efficient Shape Descriptor for an Advanced Weed Type Classification .... (Adil Tannouche) 


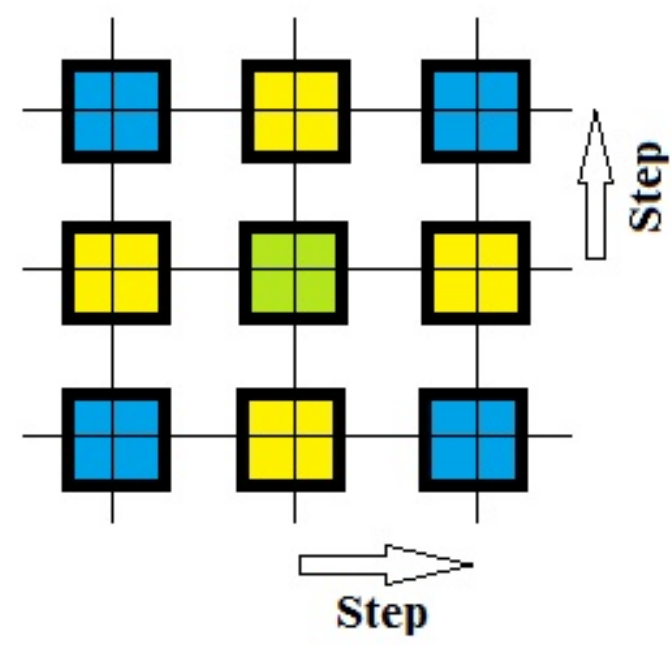

Figure 3. Descriptor structure

The proposed descriptor calculates two numbers of adjacencies between a given original pixel (C0) and their adjacent pixels (CA, such as $\mathrm{A}=1,2,3,4,5,6,7,8)$. The first is the number of horizontal and vertical adjacencies $\left(\mathrm{N}_{\mathrm{HV}}\right)$, the second is the number of diagonal adjacencies $\left(\mathrm{N}_{\mathrm{D}}\right)$. The adjacency (Adj) is the result of the binary operator XNOR between the original cell and an adjacent cell as followings:

$$
\operatorname{Adj}(\mathrm{CA})=\mathrm{C} 0(\mathrm{XNOR}) \mathrm{CA}
$$

Thereby, in a binary image, the adjacency numbers for a given original pixel $\mathrm{C}(\mathrm{x}, \mathrm{y})$ are calculated according to the following formulas:

$$
\begin{aligned}
& \mathrm{N}_{\mathrm{HV}}=\operatorname{Adj}(\mathrm{C}(\mathrm{x} \text {-step, } \mathrm{y}))+\operatorname{Adj}(\mathrm{C}(\mathrm{x}+\text { step, } \mathrm{y}))+\operatorname{Adj}(\mathrm{C}(\mathrm{x}, \mathrm{y} \text {-step }))+\operatorname{Adj}(\mathrm{C}(\mathrm{x}, \mathrm{y}+\text { step })) \\
& \mathrm{N}_{\mathrm{D}}=\operatorname{Adj}(\mathrm{C}(\mathrm{x} \text {-step,y-step }))+\operatorname{Adj}(\mathrm{C}(\mathrm{x} \text {-step,y+step }))+\operatorname{Adj}(\mathrm{C}(\mathrm{x}+\text { step,y-step })) \\
& +\operatorname{Adj}(\mathrm{C}(\mathrm{x}+\text { step,y+step }))
\end{aligned}
$$

Applying this descriptor on all pixels belonging to one particular object, allow to distinguish between shapes with rounded and filled morphology (Dicot) and others shapes with a long and thin morphology (Monocot).

The Step variable must then be adapted to the resolution of the object to describe. Indeed, a small value retains only noise, while too large value encompasses the object without defining its characteristics. Good results are obtained using the following empirical formula:

$$
S t e p=\sqrt{\frac{2}{18}}
$$

Where $\mathrm{S}$ is the area (in pixels) of the object to describe.

\subsection{Implementation}

In digital image segmentation applications, clustering technique is used to segment regions of interest and to detect borders of objects in an image [10]. The gradient magnitude and coherence is used to segment fingerprint image [11]. In our application, the obtained image is segmented in order to isolate the vegetation of the rest of the scene. According to [12], this operation is effectively carried out by thresholding the image given by the following formula:

$$
\text { Gray }=\mathrm{r} \times \mathrm{R}+\mathrm{g} \times \mathrm{G}+\mathrm{b} \times \mathrm{B}
$$

With: $\mathrm{r}=-0.884, \mathrm{~g}=1.262$ and $\mathrm{b}=-0.311$.

Where R, G and B represent red, green and blue components of each pixel. Thus, the pixels related to the vegetation are obtained for: Gray $>30$ (see Figure 4). 
The application of descriptor is realized on the segmented image by assuming a [32×32] pixels sliding window. Then, the results returned by the descriptor are classified according to DFA discrimination function (6). A majority of votes provided by the sliding window obtains the final classification of a region in the image.
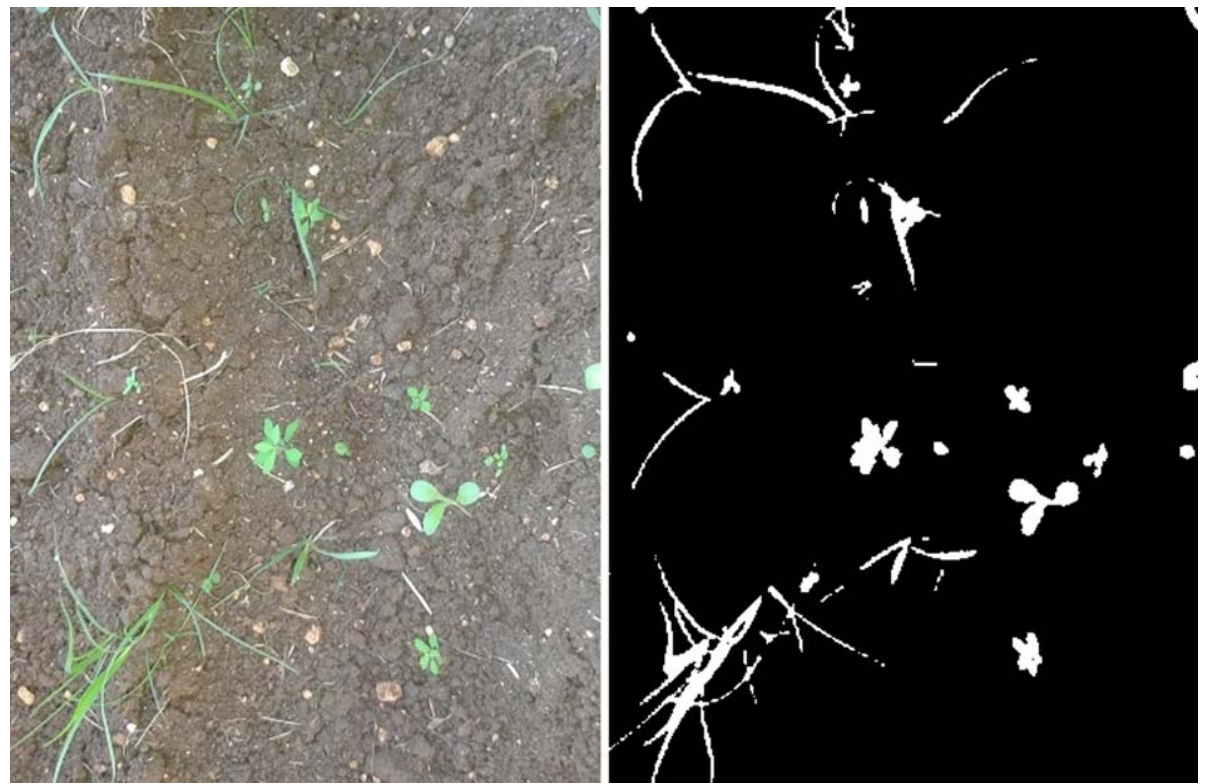

Figure 4. Segmentation Vegetation / ground, vegetation and the rest of the scene are represented respectively by white and black pixels

\section{RESULTS AND DISCUSSIONS}

The Local Binary Pattern (LBP) [13] inspires our descriptor. It allows describing the size and the morphological form of weeds by two integers $\mathrm{N}_{\mathrm{D}}$ and $\mathrm{N}_{\mathrm{HV}}$. To assess and evaluate its performance, we designed a database constituted of 40 images of the most frequent weeds. Then, each image is shifted and rotated randomly to increase the size of our database. Finally, these images are standardized to the working size [32*32], For DFA method, more than 100 subjects are suggested, but according to [14] the general rule is to have a ratio of 10 subjects per variable inserted in the analysis. For a total of 86 images, we used half of the images for learning and the other half to the test. The procedure of DFA was performed by means of XLSTAT software.

DFA is probably the most frequently used supervised pattern recognition method and the beststudied one [15]. DFA is based on the determination of discriminant functions, which maximize the ratio of between-class variance and minimize the ratio of within-class variance. As in PCA, this technique is a factorial method. In fact, using this method, data are separated in $k$ a priori defined classes. The objective sought using DFA is to investigate if the variables $\mathrm{N}_{\mathrm{D}}$ and $\mathrm{N}_{\mathrm{HV}}$ are sufficient or not to allow a good a posteriori classification of data in their a priori groups. The following tables (Table 1 and 2) show respectively the Wilks' Lambda test (Rao approximation) and the Bartlett's test of sphericity:

Table 1.Wilks' Lambda test (Rao approximation)

\begin{tabular}{cc}
\hline Lambda & 0,448 \\
F (Valeur observée) & 24,669 \\
F (Valeur critique) & 3,232 \\
DDL1 & 2 \\
DDL2 & 40 \\
p-value & $<0,0001$ \\
alpha & 0,05 \\
\hline
\end{tabular}

Wilks' Lambda test interpretation :
Table 2. Sphericity Bartlett's test

\begin{tabular}{cc}
\hline $\mathrm{Khi}^{2}$ (Valeur observée) & 55,95 \\
$\mathrm{Khi}^{2}$ (Valeur critique) & 3,841 \\
DDL & 1 \\
p-value & $<0,0001$ \\
alpha & 0,05 \\
\hline
\end{tabular}

A Fast and Efficient Shape Descriptor for an Advanced Weed Type Classification .... (Adil Tannouche) 
H0: The mean vectors of the two classes are equal.

Ha: At least one of the mean vectors is different from another.

Since the calculated $p$-value is less than the significance level of alpha $=0.05$, one must reject the null hypothesis $\mathrm{H} 0$, and retain the alternative hypothesis Ha. The risk of rejecting the null hypothesis $\mathrm{H} 0$ when it is true is less than $0.01 \%$.

Sphericity Bartlett's test interpretation:

H0: There is no significantly different correlation of 0 between variables.

Ha: At least one of the correlations between the variables is significantly different from 0 .

Since the calculated $p$-value is less than the significance level of alpha $=0.05$, one must reject the null hypothesis $\mathrm{H} 0$, and retain the alternative hypothesis Ha. The risk of rejecting the null hypothesis $\mathrm{H} 0$ when it is true is less than $0.01 \%$.

The canonical discriminant functions returned by DFA as:

$$
F 1=0.013 \mathrm{~N}_{\mathrm{HV}}-0.01 \mathrm{~N}_{\mathrm{D}}-0.96
$$

This function is used to classify the weeds into two classes Monocot and Dicot (see Figure 5). The classification quality is shown by the ROC curve (see Figure 6).

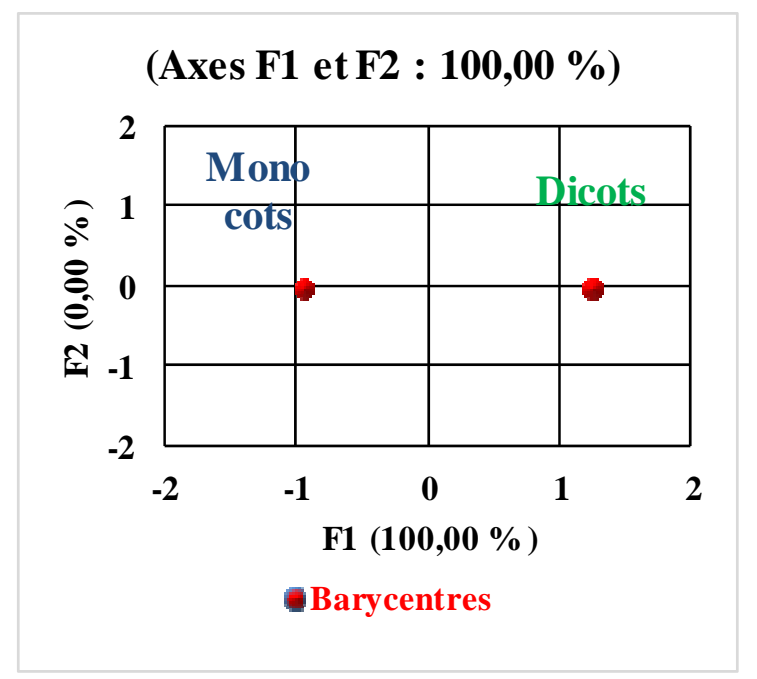

Figure 5. Weed classification according to canonical discriminant functions returned by DFA

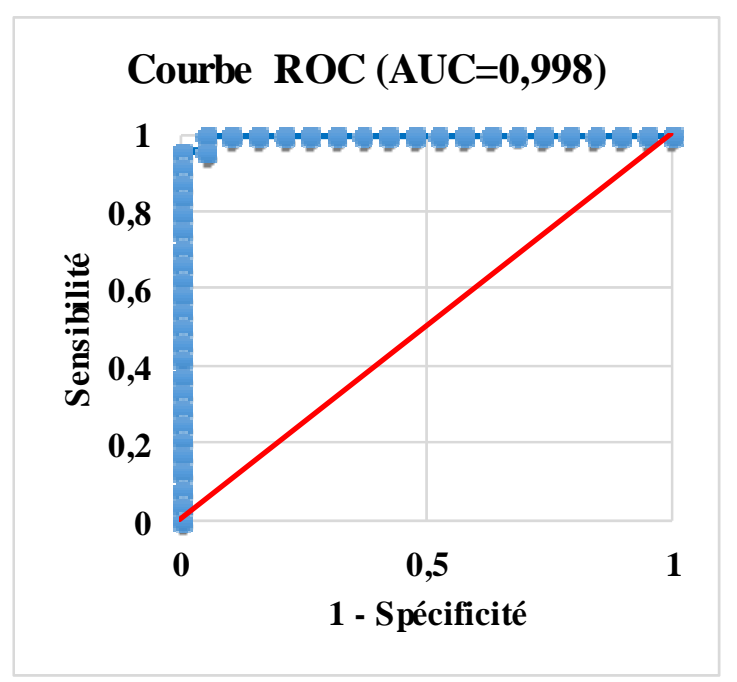

Figure 6. The ROC curve : sensitivity / specificity report.

Applying DFA on database images, a good separation between weeds species was obtained. (Figure 5) shows how the first DFA function discriminate among clusters. DFA model was cross-validated using leave-one-out approach. An accuracy of $94.74 \%$ success rate in the recognition of dicots. On the other hand, DFA classifier for the monocots has reached $95.83 \%$ of the correct classification. Total accuracy is of 95.35\% success rate (see Table 3 ).

Table 3. Confusion matrix for the results of cross-validation

\begin{tabular}{ccccc}
\hline From $\backslash$ To & Dicot & Monocot & Total & \% correct \\
\hline Dicot & 18 & 1 & 19 & 0,9474 \\
Monocot & 1 & 23 & 24 & 0,9583 \\
Total & 19 & 24 & 43 & 0,9535 \\
\hline
\end{tabular}

In addition, the main advantage of our descriptor resides in its speed (See Table 4) and ease of implementation. Indeed: Step is the only parameter to adjust. Hence, the descriptor presents robustness against the brightness change, rotation and translation.

In this table, we can clearly notice that our descriptor has a low computational cost. In practice, in precision agriculture, a scene is never covered with vegetation to $100 \%$. The experiments were conducted on 
a computer with i5 processor at $2.2 \mathrm{GHz}$ with 4 GOctets DDR4 ram. The execution time is less than $5.10^{-3} \mathrm{~s}$ by image.

Table 4. Number of possible comparisons depending on the size of the sliding window

\begin{tabular}{ccc}
\hline \multicolumn{2}{c}{ Window Size } & Number of comparisons \\
\hline Height & Width & $\mathrm{H} \times \mathrm{L} \times 8$ \\
$\mathrm{H}$ & $\mathrm{L}$ & 8192 \\
32 & 32 & 2457600 \\
\hline
\end{tabular}

This allows our descriptor to work at real time with a standard value of 25 frames per second. In practice, a majority of votes provided by the sliding window obtains the final classification of a region in the image. The results of our experiment are very promising. We got a correct classification rate of around $85 \%$ on a set of 50 images analyzed. Monocotyledonous weeds have been classified correctly up to $90 \%$ of cases, while the dicotyledonous weeds have been recognized to about $80 \%$. Figure 7 shows an example of processing performed by our approach, monocotyledonous weeds are marked in blue and dicotyledonous weeds are marked in red. This figure also shows two errors out of 26 classifications.

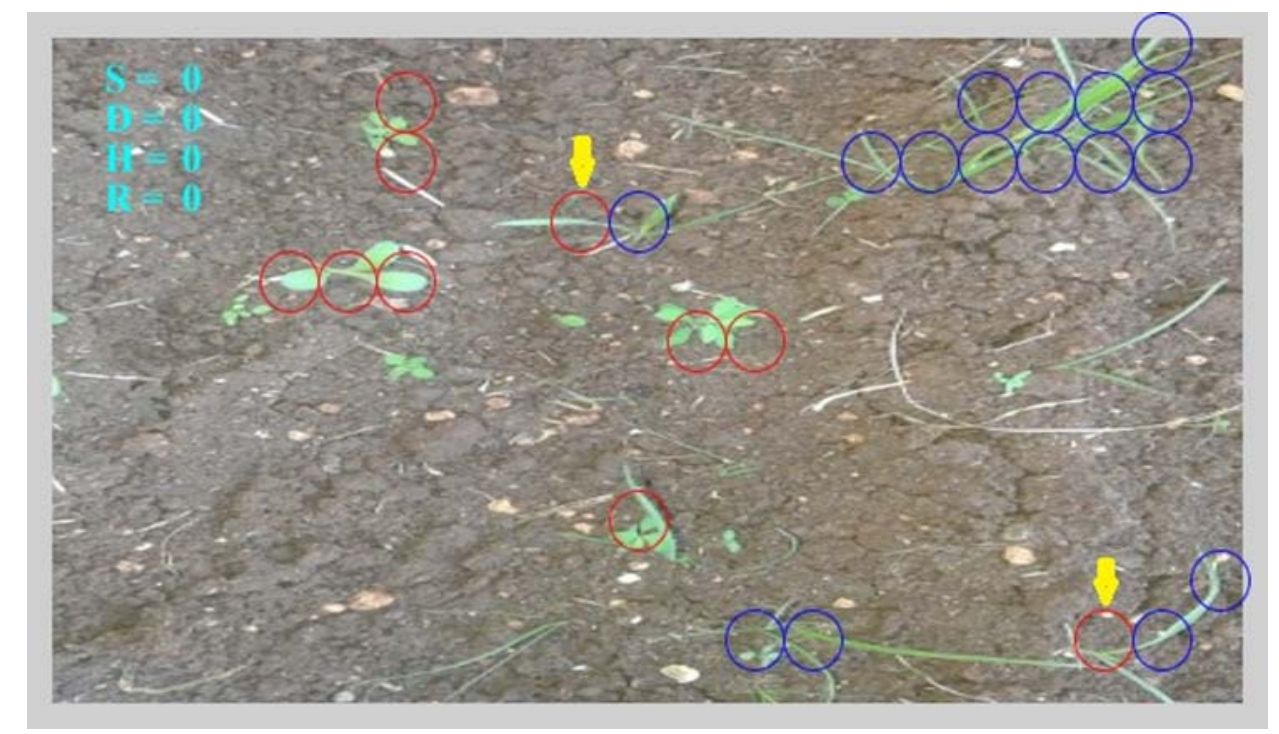

Figure 7. Classification results: Monocotyledonous in blue, dicotyledonous in red and classification errors

\section{CONCLUSION}

We achieved a system for discriminating between monocotyledonous and dicotyledonous weeds species. This system is based on our adjacencies descriptor designed for this purpose. This is a robust binary descriptor, fast and easy to use. The results obtained were very satisfactory for a fast execution time of the order of 25 frames per second. We plan to improve these results by other adopters of learning and classification methods. In the light of these results, the proposed classification systems represent an excellent, fast and efficient shape descriptor for an advanced weed type classification approach.

\section{REFERENCES}

[1] M. M. Siddiqi, et al., "A real time specific weed discrimination system using multi-level wavelet decomposition," Int. J. Agric. Biol., vol/issue: 11(5), pp. 559-565, 2009.

[2] A. Tannouche, et al., "A real time efficient management of onions weeds based on a multilayer perceptron neural networks technique," Intl J Farm \& Alli Sci., vol/issue: 4(2), pp. 161-166, 2015.

[3] L. Longchamps, et al., "Discrimination of corn, grasses and dicot weeds by their UV-induced fluorescence spectral signature," Precision Agric, vol. 11, pp. 181-197, 2010, DOI: 10.1007/s11119-009-9126-0.

[4] X. Hadoux, et al., "Weeds-wheat discrimination using hyperspectral imagery," CIGR-Ageng 2012, International Conference on Agricultural Engineering, Valencia, Spain, pp. 6, 2012. 
[5] H. T. Sogaard, "Weed classification by active shape models," Biosystems Engineering, vol/issue: 91(3), pp. 271$281,2005$.

[6] A. Tannouche, et al., "A fast and efficient approach for weeds identification using Haar-like features," AmericanEurasian Journal of Sustainable Agriculture, vol/issue: 9(4), pp. 44-48, 2015.

[7] A. Tellaechea, et al., "A computer vision approach for weeds identification through Support Vector Machines," Applied Soft Computing, vol. 11, pp. 908-915, 2011.

[8] P. J. Herrera, et al., "A novel approach for weed type classification based on shape descriptors and a fuzzy decision-making method," Sensors, vol. 14, pp. 15304-15324, 2014. DOI: 10.3390/s140815304.

[9] M. K. Hu, "Pattern recognition by moment invariants," Proc. IRE (Correspondence), vol. 49, pp.1428, 1961.

[10] J. Harikiran, et al., "Multiple feature fuzzy c-means clustering algorithm for segmentation of microarray images," International Journal of Electrical and Computer Engineering, vol/issue: 5(5), pp. 1045-1053, 2015.

[11] Saparudin, et al., "Segmentation of fingerprint image based on gradient magnitude and coherence," International Journal of Electrical and Computer Engineering, vol/issue: 5(5), pp. 1202-1215, 2015.

[12] X. P. Burgos-Artizzu, et al., "Real-time image processing for crop/weed discrimination in maize fields," Comput. Electron. Agric., vol. 75, pp. 337-346, 2011.

[13] T. Ojala, et al., "A comparative study of texture measures with classification based on feature distributions," Pattern Recognition, vol/issue: 19(3), pp. 51-59, 1996.

[14] J. F. Hair, et al., "Multivariate Data Analysis," 5th ed., Prentice-Hall, Upper Saddle River, NJ, 1998.

[15] D. F. Morrison, “Multivariate Statistical Methods," McGraw-Hill, Singapore, 2nd edition, 1988.

\section{BIOGRAPHIES OF AUTHORS}

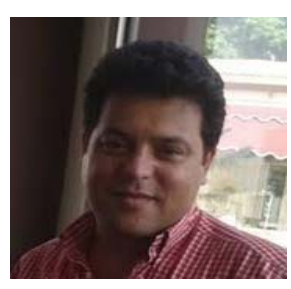

Adil Tannouche is currently a $\mathrm{PhD}$ student at the Laboratoire d'Etude des Mateériaux Avanceés et Applications, Moulay Ismail University, Faculty of Sciences in Meknes, Morocco. His research interests are focused in machine vision, artificial intelligence and theirs application in agriculture.

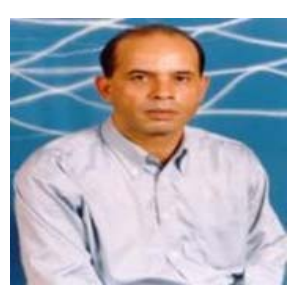

Khalid Sbai is a full professor since 2001 in Electronics. He received his M.sc. Degree in Electronics from Valencienne University (France) in 1996 and his Habilitation in Physics from Moulay Ismail University in 2008. His research interests include Structural studies, vibrational and electronic properties of carbon nanotubes

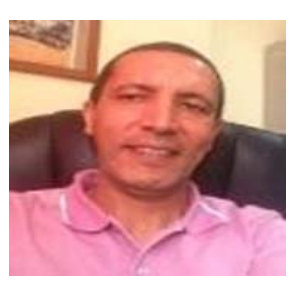

Miloud Rahmoune is a full professor at Moulay Ismail University. He received his Msc. Degree in applied mechanics from Universite' Montpellier 2 (France) and his Ph.D. degrees in Mechatronics from Universit Montpellier 2 (France) and Universite' Hassan II Mohammedia, in 1993 and 1996 respectively. His research interests include structural Dynamics, active control, and smart materials.

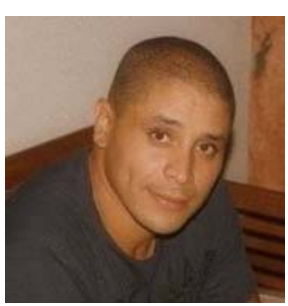

Amine Zoubir is a senior lecturer at University Moulay Ismail, Morocco. He received the MSc. Degree (Magister) in fluid mechanics from the University of Lyon 1 (France) and the Ph.D degree in Mechanics, Energetics, Civil Engineering and Acoustics of INSA Lyon (France). He is an active researcher at Thermal \& Material Research Unit (advanced materials and energy system). His area of research are focused on the numerical modeling of convective heat transfer and around the diagnosis of energy performance in buildings. 

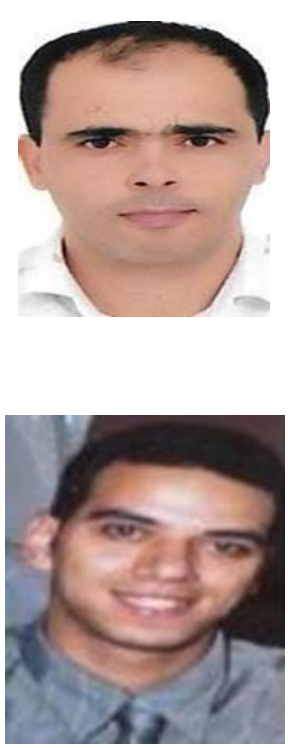

Rachid Agounoun is a senior lecturer at University Moulay Ismail, Morocco. He received the MSc. Degree (Magister) in mechanics and energetic system from the Université de Lorraine, Nancy, France and the Ph.D degree in science for engineers from the Universite' de Lorraine, Nancy, France. He is an active researcher at Thermal \& Material Research Unit (advanced materials and energy system). His area of research includes Thermal Comfort, Building Thermal Simulation, renewable energy and Porous Media.

Rachid Saadani is a senior lecturer at University Moulay Ismail, Morocco. Was born in Morocco in 1977. He received the MSc. Degree (Magister) in thermal and energetic system from the Universit Marne La Vallée, Paris Est, Paris, France and the Ph.D degree in science for engineers from the Universite' Paris Est, Créteil, Paris. He is an active researcher at Thermal \& Material Research Unit (advanced materials and energy system). His area of research includes Thermal Comfort, Building Thermal Simulation, renewable energy and Porous Media.

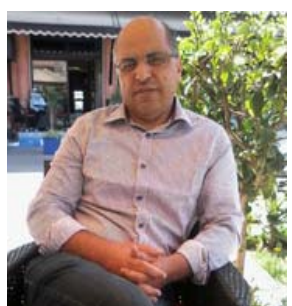

Abdelali Rahmani is a full professor at University Moulay Ismail, Morocco. He received the MSc. Degree in theoretical physics from the Universite' Montpellier 2, France and the Ph.D degree in Materials science from the the Université Montpellier 2, France. He is the Director of Laboratory of Studies of Avanced Materials and Applications). His area of research includes Computational physics and nanomaterials. 